\title{
Stochastic Models for Recognition of Occluded Objects
}

\author{
Bir Bhanu and Yingqiang Lin \\ Center for Research in Intelligent Systems, \\ University of California, Riverside, CA 92521, USA \\ bhanu@cris.ucr.edu
}

\begin{abstract}
Recognition of occluded objects in synthetic aperture radar (SAR) images is a significant problem for automatic object recognition. Stochastic models provide some attractive features for pattern matching and recognition under partial occlusion and noise. In this paper, we present a hidden Markov modeling (HMM) based approach for recognizing objects in synthetic aperture radar (SAR) images. We identify the peculiar characteristics of a SAR sensor and using these characteristics we develop feature based multiple stochastic models for a given SAR image of an object. The models exploiting the relative geometry of feature locations or the amplitude of scattering centers in SAR radar return are based on sequentialization of scattering centers extracted from SAR images. In order to improve performance under real world situations, we integrate these models synergistically using their probabilistic estimates for recognition of a particular object at a specific azimuth. Experimental results are presented using real SAR images with varying amount of occlusion.
\end{abstract}

Keywords: hidden Markov modeling, object recognition, multiple recognition models, SAR images

\section{Introduction}

One of the critical problems for object recognition is that the recognition approach should be able to handle partial occlusion of objects and spurious or noisy data [1]. In most of the object recognition approaches, the spatial arrangement of structural information of an object is the central part that offers the most important information. We suggest an object recognition mechanism that effectively makes use of the available structural information as a whole rather than viewing the spatial primitives individually. Its nondeterministic model structure makes it capable of collecting useful information from distorted or partially unreliable patterns. Many successful applications of HMM in speech recognition [2] and character recognition [3] attest to its usefulness. Thus, it is potentially an effective tool to recognize objects with partial occlusion and noise. However, the limit of traditional HMMs is that they are basically one dimensional models. In this paper we use the features based on the image formation process to encode 


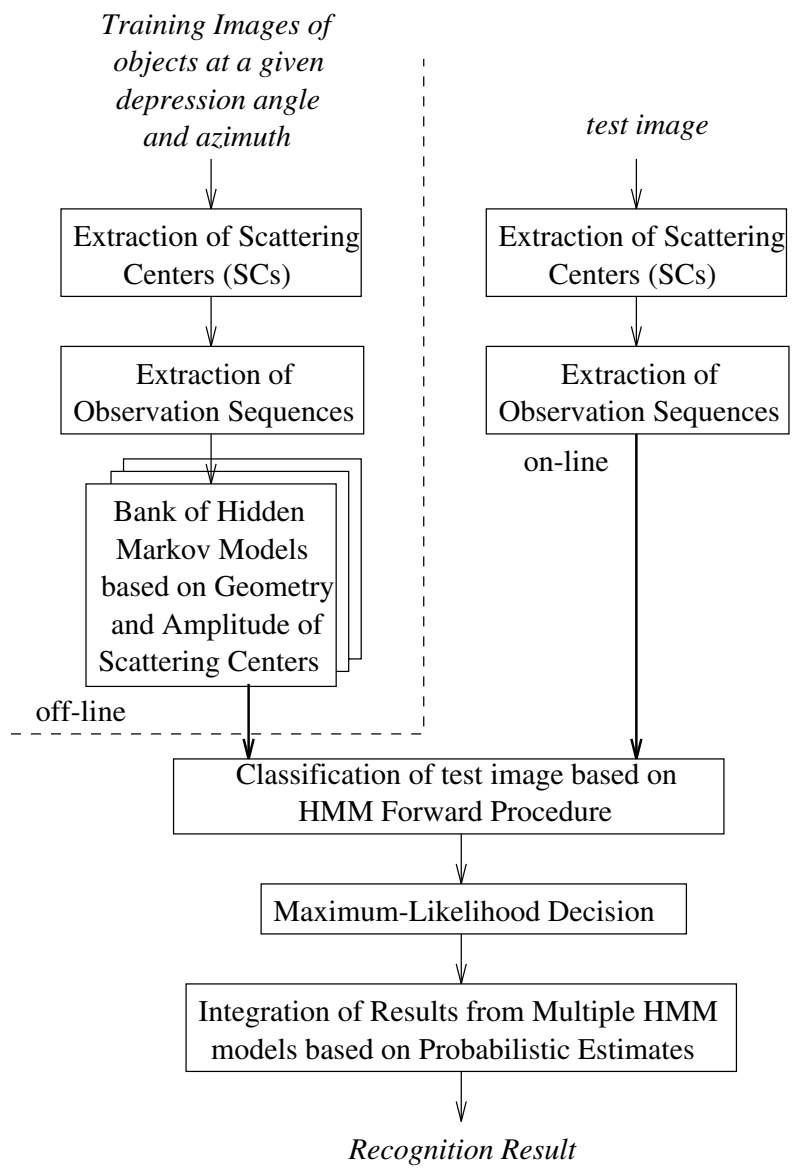

Fig. 1. The HMM-Based approach for recognition of occluded objects.

the 2-D image into 1-D sequences. We use information from both the relative positions of the scattering centers and their relative magnitude in SAR images to address the fundamental issues of building object models and using them for robust recognition of occluded objects.

\section{Technical Approach}

Figure 1 provides an overview of the HMM based approach for recognition of occluded objects in SAR imagery. During an off-line phase, scattering centers are extracted from SAR images by finding local maxima of intensity. Both locations and magnitudes of these peak features are used in the approach. These features are viewed as emitting patterns of some hidden stochastic process. Multiple observation sequences based on both the relative geometry and amplitude 
of the SAR return signal are used to build the bank of stochastic models. These models provide robust recognition in the presence of severe occlusion and unstable features caused by scintillation phenomena where some of the features may appear/disappear at random in an image. At the end of the off-line phase, hidden Markov recognition models for various objects and azimuths are obtained. Similar to the off-line phase, during the on-line phase features are extracted from SAR images and observation sequences based on these features are matched by the HMM forward process with the stored models obtained previously. Maximum likelihood decision is made on the classification results. Now the results obtained from multiple models are combined in a voting kind of approach that uses both the object, azimuth label and its probability of classification. This produces a rank ordered list of classifications of the test image and associated confidences.

\subsection{Related Work}

Preliminary work using HMM models for recognition of objects in infrared imagery is described by Burger and Bhanu [4]. Kottke et al. [5] use Radon transform in conjunction with HMM for representation and segmentation of nonoccluded objects in SAR images. Fielding and Ruck [6] have used HMM models for spatialtemporal pattern recognition to classify moving objects in image sequences. Rao and Mersereau [7] have attempted to merge HMM and deformable template approaches for image segmentation. Template matching [8] and major axis [9] based approaches have been used to recognize and index objects in SAR images, however, they are not suitable to recognize occluded objects. Jones and Bhanu [10] use a geometric hashing kind of approach for recognizing occluded objects in simulated SAR images [10].

\subsection{Hidden Markov Modeling Approach}

HMM is defined as a triple $\lambda=(A, B, \pi)$, where $a_{i j}$ is the probability that state $i$ transits to state $j, b_{i j}(k)$ is the probability that we observe symbol $k$ in a transition from state $i$ to state $j$, and $\pi_{i}$ is the probability of $i$ being the initial state.

Recognition Problem - Forward Procedure: The HMM provides us a useful mechanism to solve the problems we face for robust object recognition. Given a model and a sequence of observations, the probability that the observed sequence was produced by the model can be computed by the forward procedure. Suppose we have a $\operatorname{HMM} \lambda=\{A, B, \pi\}$ and an observation sequence $y_{1}^{T}$. We define $\alpha_{i}(t)$ as the probability that the Markov process is in state $i$, having generated $y_{1}^{t}$, i.e., $\alpha_{i}(t)=\Sigma_{j}\left[\alpha_{j}(t-1) a_{j i} b_{j i}\left(y_{t}\right)\right]$, when $t>0$, where $\alpha_{i}(0)=1$ or 0 , depending upon whether it is an initial state or not, respectively. 
The probability that the HMM stopped at the final state and generated $y_{1}^{T}$ is $\alpha_{S_{F}}(T)$. After initialization of $\alpha$, we compute it inductively. At each step the previously computed $\alpha$ is used, until $T$ is reached. $\alpha_{S_{F}}(T)$ is the sum of probabilities of all paths of length $T$.

Training Problem - Baum-Welch Algorithm: To build a HMM is actually an optimization of the model parameters so that it can describe the observation better. This is a problem of training. The Baum-Welch re-estimation algorithm is used to calculate the maximum likelihood model. But before we use the Baum-Welch algorithm, we must introduce the counterpart of $\alpha_{i}(t), \beta_{i}(t)$, which is the probability that the Markov process is in state $i$ and will generate $y_{t+1}^{T}$, i.e., $\beta_{i}(t)=\Sigma_{j}\left[a_{i j} b_{i j}\left(y_{t+1}\right) \beta_{j}(t+1)\right]$, when $0 \leq t<T, \beta_{i}(T)=1$ or 0 , depending upon whether it is a final state or not, respectively. The probability of being in state $i$ at time $t$ and state $j$ at time $t+1$ given observation sequence $y_{1}^{T}$ and the model $\lambda$ is defined as follows:

$$
\begin{aligned}
\gamma_{i j}(t) & =P\left(X_{t}=i, X_{i+1}=j \mid y_{1}^{T}\right) \\
& =\frac{\alpha_{i}(t-1) a_{i j} b_{i j}\left(y_{t}\right) \beta_{j}(t)}{\alpha_{S_{F}}(T)}
\end{aligned}
$$

Now the expected number of transitions from state $i$ to state $j$ given $y_{1}^{T}$ at any time is simply $\Sigma_{t=1}^{T} \gamma_{i j}(t)$ and the expected number of transitions from state $i$ to any state at any time is $\Sigma_{t=1}^{T} \Sigma_{k} \gamma_{i k}(t)$. Then, given some initial parameters, we could recompute $\overline{a_{i j}}$, the probability of taking the transition from state $i$ to state $j$ as:

$$
\overline{a_{i j}}=\frac{\Sigma_{t=1}^{T} \gamma_{i j}(t)}{\Sigma_{t=1}^{T} \Sigma_{k} \gamma_{i k}(t)}
$$

Similarly, $\overline{b_{i j}(k)}$ can be re-estimated as the ratio between the frequency that symbol $k$ is emitted and the frequency that any symbol is emitted:

$$
\overline{b_{i j}(k)}=\frac{\sum_{t: y_{t}=k} \gamma_{i j}(t)}{\sum_{t=1}^{T} \gamma_{i j}(t)}
$$

It can be proved that the above equations are guaranteed to increase $\alpha_{S_{F}}(T)$ until a critical point is reached, after which the re-estimate will remain the same. In practice, we set a threshold as the ending condition for re-estimation.

So the whole process of training a HMM is as follows: (1) Initially, we have only an observation sequence $y_{1}^{T}$ and blindly set $(A, B, \pi)$. (2) Use $y_{1}^{T}$ and $(A, B, \pi)$ to compute $\alpha$ and $\beta$ (3) Use $\alpha$ and $\beta$ to compute $\gamma$. (4) Use $y_{1}^{T}$, $(A, B, \pi), \alpha, \beta$ and $\gamma$ to compute $A$ and $B$. Go to step 2 . 


\subsection{SAR Features}

The features of SAR images we used are the scattering centers (local maxima), and the magnitude of these scattering centers. Unlike the visible images, SAR images are extremely sensitive to slight changes in viewpoint (azimuth and depression angle) and are not affected by scale. As a result, the magnitude and location of scattering centers are very sensitive to rotational transformation. It is observed that typically less than $1 / 3$ of the scattering center locations remain unchanged on XPATCH simulated SAR data [10]. Similar results are obtained with real SAR data at one foot resolution. Therefore, to recognize occluded objects, we need to get SAR images of an object at a given depression angle under various finely sampled azimuth angles. Ideally, we can have $360 \mathrm{SAR}$ images of an object with one image corresponding to one and only one azimuth angle between $0^{\circ}$ and $359^{\circ}$. Thus, we treat an object under different azimuth angles separately and build a model for each azimuth angle based on the SAR image taken under this azimuth angle.

Note that when building model for an object azimuth, a single model is inadequate because of noise, articulation, occlusion, etc. Therefore, to increase robustness, we build multiple HMM models for a given object at a specific azimuth, as discussed in the next section.

\subsection{Extraction of Observation Sequences}

There are many ways to choose observation sequences, but we want to use information from both the magnitude and the relative spatial location of the scattering centers extracted from a SAR image. Also the sequentialization method should not be affected by distortion, noise, or partial occlusion and should be able represent the image efficiently. Based on the above considerations, we employ two approaches to obtain the sequences. We assume that the scattering centers have been sorted according to their magnitude in a descending order. Note that sequences $O_{1}$ and $O_{2}$ are of length n, whereas the $O_{3}, O_{4}, O_{5}$ are of length $(\mathrm{n}-1)$.

- Sequences based on amplitudes: $O_{1}=\left\{M a g_{1}, M_{a} g_{2}, \ldots, M a g_{n}\right\}$, where $M a g_{i}$ is the amplitude of $i$ th scattering center.

- Sequences based on relative geometrical relationships:

$$
\begin{aligned}
& O_{2}=\{d(1,2), d(2,3), \ldots, d(n, 1)\} \\
& O_{3}=\{d(1,2), d(1,3), \ldots, d(1, n)\} \\
& O_{4}=\{d(2,1), d(2,3), \ldots, d(2, n)\} \\
& O_{5}=\{d(3,1), d(3,2), \ldots, d(3, n)\},
\end{aligned}
$$

where $d(i, j)$ is the Euclidean distance between scattering centers $i$ and $j$.

Figure 2 gives an example to illustrate how we get the sequences. Sequence $O_{1}$ is obtained by sorting the scattering centers by their magnitude (no location information). It captures the characterisitcs of the magnitudes of the scattering centers. Sequences $\mathrm{O}_{2}$ through $\mathrm{O}_{5}$ are obtained based on the relative locations 


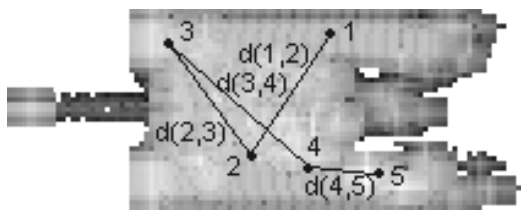

Fig. 2. Example of an observation sequence superimposed on an image of a tank.

of the scattering centers and magnitude of the scattering centers is not used. These four sequences capture the geometric structure of the scattering centers and measure the relative distances between the scattering centers. The actual distances between scattering centers are determined by the resolution of the SAR image and they are independent of the distance between the sensor and the target. Thus, sequences $\mathrm{O}_{2}$ through $O_{5}$ are not dependent on scale.

In experiments described in Section 3, we only consider the top 30 scattering centers (sorted in descending order of their magnitude). This is because we expect that the scattering centers with larger magnitude are relatively more stable than the weaker ones.

\subsection{Integration of Results from Multiple Sequences}

Since not all models based on various sequences for a particular object and azimuth will provide optimal recognition performance under occlusion, noise, etc., we improve the recognition performance by combining the results obtained from multiple kinds of models.

We have developed a voting-like method to integrate the results from models based on a given number of sequences. The algorithmic steps are:

(1) For each test image, we collect the $\mathrm{N}$ highest possibilities in the test results corresponding to each of the sequences. Each possibility is the probability that the test image is the image of that object at that azimuth.

(2) A normalization is done to the $\mathrm{N}$ probabilistic estimates corresponding to each of the sequences so that estimates from different sequences can be compared.

(3) We draw a histogram with probability vs. object and azimuth for the results obtained in step (2). We pick up the object with the highest frequency in the histogram.

(4) If the object associated with the highest frequency in the histogram is the same as the groundtruth, we count it as one correct recognition. 


\subsection{Recognition Procedure}

The recognition procedure is described as follows:

(1) Loop (2), (3) for all the testing observation sequences.

(2) Loop (3) for all the models in the model base.

(3) Feed the observation sequence into the model, $(A, B, \Pi)_{\left(M_{i}^{*}, a_{j}^{*}\right)}$. Use Forward algorithm to compute the probability that this sequence is produced by this model.

(4) The model with maximum probability of an observation sequence is selected as the best match.

(5) For each testing sequence, perform integration on the results of individual sequences.

(6) For each testing sequence, obtain final recognition result by selecting the object class with the highest frequency of occurrence.

\section{Experiments}

- Data: We use MSTAR public real SAR images (at one foot resolution and depression angle $15^{\circ}$ ) of 2 objects (T72 tank with serial number \#a64 and ZSU tank with serial number \#d08). Ideally, we can have 360 object models for each azimuth for each object. However, we don't have 360 SAR images for each object in the MSTAR data set. For the T72 tank, there are 288 images available for different azimuths. Also for the ZSU tank, 288 images are available. Thus, each object consists of 288 azimuths (or aspects) which we call object models. Each object model consists of HMM models based on observation sequences $\left(O_{1}\right.$ to $\mathrm{O}_{5}$ ). We extract 30 scattering centers with largest magnitudes from each SAR image. Figure 3 shows some examples of SAR imagery, region of interest and scattering centers superimposed on the SAR image. ROIs are obtained using a dilation/erosion process automatically.

We consider the occlusion to occur possibly from 9 different directions (center, 4 sides and 4 corners of the image). Scattering centers being occluded are not available. Moreover, we add back into the image at random locations a number of spurious scattering centers, equal to the number of occluded scatterers, of random magnitude. For example, for $30 \%$ occlusion, we remove 9 scattering centers from the center of one object or from one particular direction and add randomly 9 spurious scattering centers back into the image. We compute the observation sequences based on the scattering centers available after the occlusion process has taken place.

Training Data: We generate 91 training sequences of each type $\left(O_{i}\right)$ from each SAR image. The first one is obtained from the original SAR image without occlusion. Then we occlude the SAR image from 9 directions. For each direction, the occlusion level is $5 \%$ and $10 \%$. For each occlusion level, we extract 5 training observation sequences. So 91 sequences are generated from each image. We have 


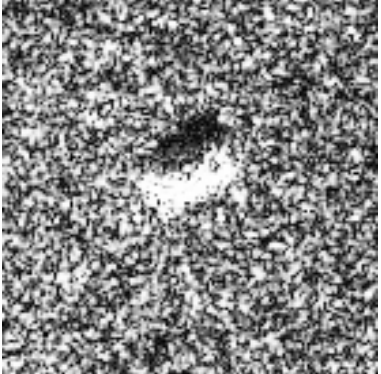

(a) SAR image of a T72 tank

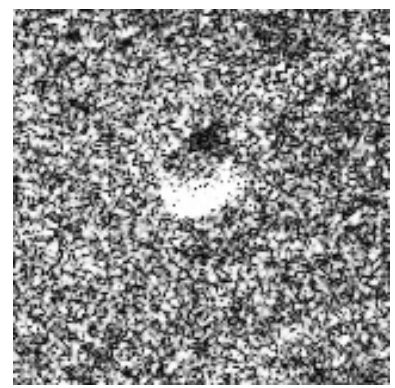

(c) SAR image of a ZSU tank

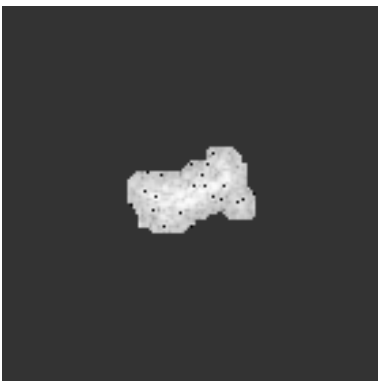

(b) Features extracted for T72 tank

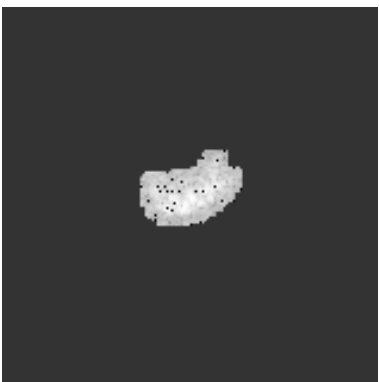

(d) Features extracted for ZSU tank

Fig. 3. Real SAR images and region of interests (ROIs) (with peaks shown as black dots superimposed on the ROI) for T72 tank \#a64 and ZSU tank \#d08.

two objects and 288 SAR images of each object, thus the number of training sequences of each type $\left(O_{i}\right)$ is 52,416 . Since there are 5 kinds of observation sequences, the total number of sequences is 262,080 .

Testing Data: From each SAR image, we generate 37 testing sequences of each type $\left(O_{i}\right)$. The first one is obtained from the original SAR image without occlusion. Then we occlude the SAR image from 9 directions. For each direction, the occlusion level is from $20 \%$ to $50 \%$ with $10 \%$ increment. For each occlusion level, we extract only 1 testing sequence. Thus, we have total 21,312 testing sequences. In our experiment, we require that the occlusion level be $20 \%, 30 \%$, $40 \%$ and $50 \%$. That is, the numbers of occluded scattering centers are $6,9,12$ and 15 respectively. Also, when testing, we only use the sequences which are obtained when the occlusion was from direction 1 , which is the direction from the right side of the image. So, for each occlusion level, we have 576 testing 
sequences. Since there are 5 kinds of observation sequences, the total number of sequences for each occlusion level is 2880 .

- Training: We performed experiments to choose the optimum of number of states and number of symbols of the HMM. We find that with the increase in the number of states and symbols, recognition performance increases. Considering both the recognition performance and the computation cost, we choose 8 states and 32 symbols as the optimal number of states and symbols for our HMM models. Using the algorithm presented above we built recognition models. For one sequence type we have 576 (288 azimuths $\times 2$ object classes) HMM models. Since we have defined five kinds of observation sequences for each image $\left(\mathrm{O}_{1}, \mathrm{O}_{2}, \mathrm{O}_{3}, \mathrm{O}_{4}, \mathrm{O}_{5}\right)$, we get models based on each kind of observation sequence.

- Testing Results: During the testing phase, for a given observation sequence type $\left(\mathrm{O}_{1}, \mathrm{O}_{2}, \mathrm{O}_{3}, \mathrm{O}_{4}, \mathrm{O}_{5}\right)$, each of the 576 testing sequences is tested against all models (576 models: 2 objects, each has 288 models for each azimuth angle available). An object model represents the object at a particular azimuth angle. Here we consider only the kind of an object, which we call ID, we count a recognition result as a correct recognition if the HMM model with the maximum probability is associated with the object from which the testing sequence was extracted. We do not consider the corresponding azimuth angles of the HMM model and testing sequence.

The recognition results are shown in Table 1 and 2 . These results are satisfactory at $30 \%-40 \%$ of occlusion. Table 1 shows the results when we combine the results of all five sequences $\left(O_{1}\right.$ to $\left.O_{5}\right)$. Table 2 shows the results when only sequences $O_{1}$ and $O_{2}$ are used in integration. From these two tables, we can see that the performance degrades as the occlusion level increases. The results of Table 2 based on $O_{1}$ and $O_{2}$ are slightly better than the results of Table 1 based on all the five sequences $\left(O_{1}\right.$ to $\left.O_{5}\right)$. There are two reasons for this degradation in performance.

- First, the results from sequences $O_{3}, O_{4}$, and $O_{5}$ are less reliable than others. Each of these sequences measures distances from a specific scattering center, for example, $\mathrm{O}_{3}$ measures distances of other scattering centers from (reference) scattering center $1, \mathrm{O}_{4}$ measures distances of other scattering centers from scattering center 2, and so on. At lower rates of occlusion, there is lower probability of prominent scattering centers (with lower numbers) to get occluded than at higher levels of occlusion. As a result, at higher level of occlusion, the performance of each of the sequences $\mathrm{O}_{3}, \mathrm{O}_{4}$, and $\mathrm{O}_{5}$ deteriorates (e.g., if the first scattering center is occluded, then the entire sequence $\mathrm{O}_{3}$ is subject to error), which is reflected in the integration results. An alternative is to use all the scattering centers as reference points to build models to achieve increased performance at the expense of increased computation, not just the top three scattering centers that we used in sequences $\mathrm{O}_{3}, \mathrm{O}_{4}$, and $\mathrm{O}_{5}$. 
- Second, the relative distances in $O_{3}$ to $O_{5}$ are expected to be longer than those in $\mathrm{O}_{2}$. As a result of occlusion, the longer distances are more strongly affected than the relatively shorter distances in $\mathrm{O}_{2}$, which measures the relative distances between successive scattering centers.

\section{Conclusions}

We have presented a novel conceptual approach for the recognition of occluded objects in real SAR images. The approach uses multiple HMM based models for various observation sequences that are chosen based on the SAR image formation and account for both the geometry and magnitude of SAR image features. We have demonstrated that HMM approach makes use of the available structural information to solve the problem caused by occlusion and noise. It takes the spatial arrangement of structural information as a whole and is able to collect useful information from distorted or partially unreliable patterns. The results generally meet the desired goals at 30\% - 40\% occlusion.

Table 1. Results of Integration (Sequences $O_{1}$ to $O_{5}$ )

\begin{tabular}{|c||c|c|c|c||c|}
\hline \multirow{2}{*}{\multicolumn{1}{|c||}{ Occlusion Level }} & \multicolumn{4}{c||}{ \# of Correct Recognition } & \# of test \\
\cline { 2 - 5 } & $20 \%$ & $30 \%$ & $40 \%$ & $50 \%$ & sequences \\
\hline \hline T72 Tank & $280(97.2 \%)$ & $233(80.9 \%)$ & $200(69.4 \%)$ & $164(56.9 \%)$ & 288 \\
\hline ZSU Tank & $283(98.3 \%)$ & $224(77.8 \%)$ & $190(66.0 \%)$ & $171(59.4 \%)$ & 288 \\
\hline \hline
\end{tabular}

Table 2. Results of Integration (Sequences $O_{1}$ and $O_{2}$ )

\begin{tabular}{|c||c|c|c|c||c|}
\hline \multirow{2}{*}{ Occlusion Level } & \multicolumn{4}{|c||}{ \# of Correct Recognition } & \# of test \\
\cline { 2 - 5 } & $20 \%$ & $30 \%$ & $40 \%$ & $50 \%$ & sequences \\
\hline \hline T72 Tank & $284(98.6 \%)$ & $249(86.5 \%)$ & $208(72.2 \%)$ & $186(64.6 \%)$ & 288 \\
\hline ZSU Tank & $285(99.0 \%)$ & $238(82.6 \%)$ & $194(67.4 \%)$ & $175(60.8 \%)$ & 288 \\
\hline \hline
\end{tabular}

Acknowledgments: This research is supported in part by grant F49620-97-10184. The contents and information do not necessarily reflect the positions and policies of the U.S.Government. 


\section{References}

1. B. Bhanu, D.E. Dudgeon, E.G. Zelnio, A. Rosenfeld, D. Casasent and I.S. Reed. Introduction to the special issue on automatic target recognition. IEEE Transactions on Image Processing, Vol. 6, No. 1, January 1997

2. L.R. Rabiner and B.H. Juang. An introduction to hidden Markov models. IEEE ASSP Magazine, 3(1):4-16, Jan 1986.

3. O.E. Agazzi and S.S. Kuo. Hidden Markov model based optical character recognition in the presence of deterministic transformations. Pattern Recognition, 26(12):18131826, November 1993.

4. W. Burger and B. Bhanu. Signal-to-symbol conversion for structural object recognition using hidden Markov models. Proc. ARPA Image Understanding Workshop, Monterey, CA, November 13-16, pp. 1287-1291, 1994.

5. D.P. Kottke et al. A design for HMM-based SAR ATR. SPIE Conf. on Algorithm for Synthetic Aperture Radar Imagery V, Vol. 3370, pp. 541-551, April 1998.

6. K.H. Fielding and D.W. Ruck. Spatio-temporal pattern recognition using hidden Markov models. IEEE Trans. Aerospace and Electronic Systems, 31(4):1292-1300, 1995.

7. R.R. Rao and R.M. Mersereau. On merging hidden Markov models with deformable templates. In Proc. International Conf. on Image Processing, pages 556-559, 1995.

8. L. Novak, G. Owirka and C. Netishen. Radar target identification using spatial matched filters. Pattern Recognition, Vol. 27, No. 4, pp. 607-614, 1994.

9. J. Yi, B. Bhanu and M. Li. Target indexing in SAR image using scattering centers and Hausdoff distance. Pattern Recognition Letters, Vol. 17, pp. 1191-1198, Sept. 1996.

10. G. Jones III and B. Bhanu. Recognition of Articulated and Occluded Objects. IEEE Transactions on Pattern Analysis and Machine Intelligence, Vol. 21, No. 7, pp. 603-613, July 1999. 